\title{
Hybrid Page Layout Analysis via Tab-Stop Detection
}

\author{
Ray Smith \\ Google Inc. 1600 Amphitheatre Parkway, Mountain View, CA 94043, USA. \\ theraysmith@gmail.com
}

\begin{abstract}
A new hybrid page layout analysis algorithm is proposed, which uses bottom-up methods to form an initial data-type hypothesis and locate the tab-stops that were used when the page was formatted. The detected tab-stops, are used to deduce the column layout of the page. The column layout is then applied in a top-down manner to impose structure and reading-order on the detected regions.

The complete $\mathrm{C}++$ source code implementation is available as part of the Tesseract open source OCR engine at http://code.google.com/p/tesseract-ocr.
\end{abstract}

\section{Introduction}

Physical Page layout analysis, one of the first steps of OCR, divides an image into areas of text and nontext, as well as splitting multi-column text into columns. This paper does not address logical layout analysis, which detects headers, footers, body text, numbered lists, and segmentation into articles.

Physical Layout Analysis is essential to enable an OCR engine to process images of arbitrary pages, such as from books, magazines, journals, newspapers, letters, and reports. Methods for physical layout analysis fall roughly into two categories:

Bottom-up methods are both the oldest [1] and more recently published $[2,3]$ methods. They classify small parts of the image (pixels, groups of pixels, or connected components), and gather together like types to form regions. The key advantage of bottom-up methods is that they can handle arbitrarily shaped regions with ease. The key disadvantage is that they struggle to take into account higher-level structures in the image, such as columns. This often leads to overfragmented regions.

Top-down methods [4] cut the image recursively in vertical and horizontal directions along whitespaces that are expected to be column boundaries or paragraph boundaries. Although top-down methods have the advantage that they start by looking at the largest structures on the page, they are unable to handle the variety of formats that occur in many magazine pages, such as non-rectangular regions and cross-column headings that blend seamlessly into the columns below.

A third type of method [5-7] is based on analysis of the whitespace in an image. This solves some of the flaws in the recursive top-down methods, by finding gaps between columns by a bottom-up analysis of the gaps, looking explicitly for white rectangles. These algorithms mostly still suffer from the problem of being unable to handle non-rectangular regions.

\section{Page layout via tab-stop detection}

When a page is laid out, either by a professional publishing system, or by a common word processor, the regions of a page are bounded by tab-stops. The margins, column edges, indentation, and columns of a table are all placed at fixed x-positions at which edges or centers of text lines are aligned vertically. Tab-stops distinguish tables from body text, and they also bound rectangular non-column elements, such as inset images and pull-out quotes.

The tab-stops in the example of Fig. 1 are the column boundaries with an additional tab-stop for the paragraph indentation that is not required for finding the

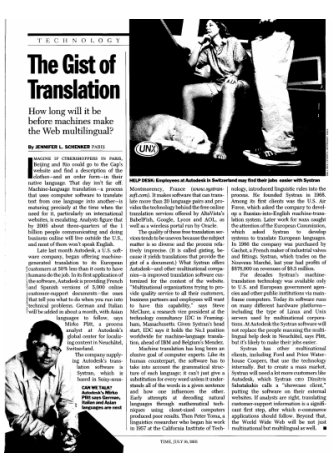

Fig.1. Input image. page layout. The non-rectangular inset image, typically, strays outside of the column boundaries.

In some sense, white rectangles match tab-stops, but white rectangles may be disrupted by background noise or background images. Also the ends of white rectangles do not match the ends of the region bounded by the tab-stops, because the white rectangles run on into the perpendicular whitespace. 
The proposed algorithm is similar to the whitespace rectangle methods in that it uses a bottom-up method to find a top-down structure, but instead of finding the space between columns, it looks for the tab-stops that mark their edges, and, through further combination of bottom-up and top-down methods, copes easily with non-rectangular regions.

There are for main phases: preprocessing, in which bottom-up morphological and connected component analysis form initial hypotheses over the local data types; bottom-up tab-stop detections; finding the columns layout; and finally applying the column layout to create an ordered set of typed regions. These phases will be detailed in sections 3-6.

\section{Preprocessing}

The aim of the preprocessing step is to identify line separators, image regions, and separate the remaining connected components into likely text components and a smaller number of uncertain type.

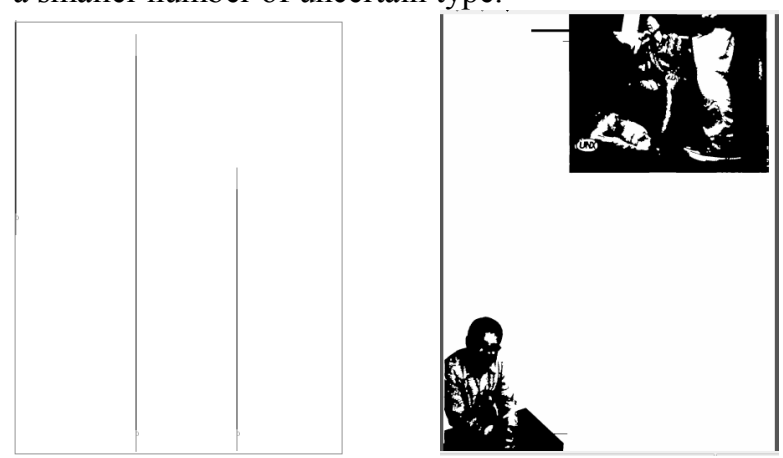

Fig.2. (a) Vertical lines, (b) Image elements.

Starting with the image of Fig. 1, the morphological processing from Leptonica [8] detects the vertical lines shown in Fig. 2(a) and the image mask shown in Fig. 2(b). These detected elements are subtracted from the input image before passing the cleaned image to connected component analysis.

The connected components (CCs) are filtered by width, $w$, and height, $h$ into small, medium, and large sizes as follows: CCs with $h<7$ (at 300ppi) are small. The $75^{\text {th }}$ percentile of the heights of the remainder, $h_{75}$, is computed, and CCs with $h<h_{75} / 2$ are small; $h>2 h_{75}$ or $w>8 h_{75}$ are large, and the rest are medium.

This filtration is important, since small CCs (noise or diacriticals) and large non-text CCs, (line drawings, logos, or frames) are likely to confuse the text-line algorithms, but large text headings are important to reading order detection. Large CCs are considered text at this stage if there is a left or right neighbor that has a similar stroke width. On "stressed" fonts, the stroke width is greater on vertical lines than on horizontal lines, so stroke width is calculated separately in both directions. Stroke width is calculated from horizontal and vertical local maxima of the distance function on the binary image of the CC. Fig. 3 shows the $\mathrm{CCs}$ are filtered as medium or large text.

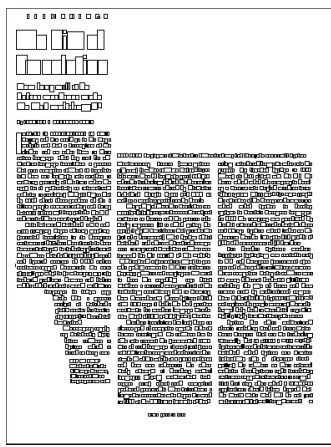

Fig.3. Filtered CCs

\section{Finding tab positions as line segments}

The process of finding tab-stop line segments has several major sub-steps: candidate tab-stop CCs that look like they may be at the edge of a text region are found and then grouped into tab-stop lines, then connections between tab-stop lines are found, enabling removal of false positives.

\subsection{Finding candidate tab-stop components}

The initial candidate tab-stop CCs are found by a radial search starting at every filtered $\mathrm{CC}$ from preprocessing. Assuming that the $\mathrm{CC}$ is at a tab-stop, the search looks for aligned neighbors and neighbors in the gutter where there should be a space. Each CC is processed independently and marked according to whether it is a candidate left tab, right tab or neither. Fig. 4(a) illustrates the candidate tab-stop CCs.

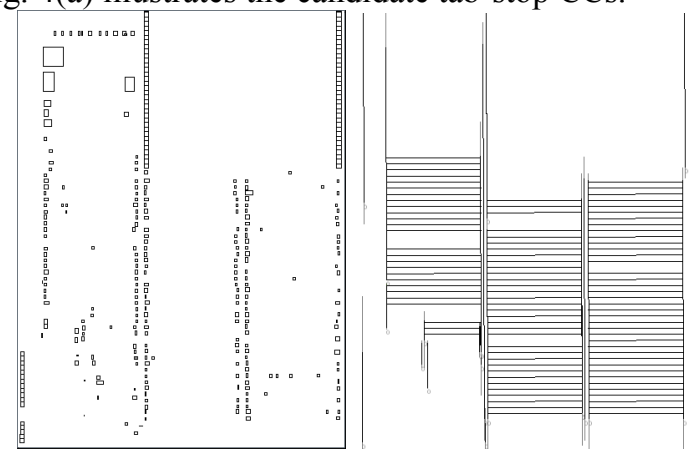

Fig.4. (a) Candidate tab-stop components (b) Fitted tab lines and traces connections.

\subsection{Grouping candidate tab components}

Candidate tab CCs are grouped into lines, and, where there are sufficiently many CCs in a group, they are kept. A least median of squares algorithm is used to fit a line to the appropriate (left or right) edge of each $\mathrm{CC}$ in a group. After finding all tab-stop line segments, all the lines are refitted to the page-mean direction, 
such that all the member tab CCs fall to one side of the line segment.

\subsection{Tracking text lines to connect tab stops}

The next step connects tab-stops by tracking text lines from one tab-stop to another. Closely adjacent, vertically overlapping CCs qualify, but large gaps cannot be jumped. Tab-stops that have a text-line connecting them are associated with each other, as being likely opposite sides of a text column. Fig. 4(b) shows the tab-stop lines and connecting text lines. Tabstop lines that have no connection are discarded.

The most frequently occurring widths of the text lines connecting tab stops are recorded for use in finding the column layout.

\subsection{Cleaning up tab stop ends}

The final step attempts to make connected tab lines end at the same y coordinate, by allowing the ends to move between the last member $\mathrm{CC}$ whose edge was used for the tab line, and the first nonmember $\mathrm{CC}$ that the line intersects. Fig. 5 shows the final tab line segments.

After construction of the tab stops, the CCs are reclassified, as "Text" or

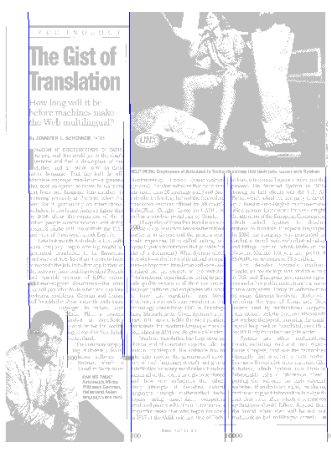

Fig.5. Cleaned tab-stops.
"Unknown" using the same text-line tracing algorithm as was used above to find connections between tab stops. If a group of CCs of significant width form a text line, then they are classified as text. Artificial image CCs of about the same size as the body-text CCd are created from the image mask from the morphological preprocessing.

\section{Finding the column layout}

The next major step is to find the column layout of the page. All the rest of the steps make use of the Column Partition (CP) objects which are created now.

Scanning the CCs from left to right and top to bottom, runs of similarly classified (text, image, or unknown) $\mathrm{CCs}$ are gathered into $\mathrm{CPs}$, subject to the constraint that no CP may cross a tab stop line. Fig. 6 shows the result of this process. A collection of CPs from a single horizontal scan are stored in a Column Partition Set (CPset).
Each CPset is potentially a division of the page into columns at that vertical position. Finding the column layout is therefore a process of finding an optimal set of CPsets that best "explains" (see below) all the CPsets on the page, but first some definitions:

A good CP either touches a tab line on both vertical edges of its bounding box, or its width is

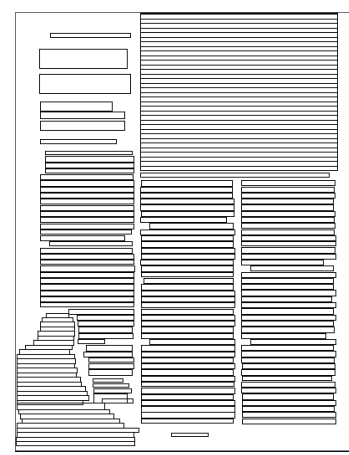

Fig.6. Column Partitions (CPs) close to a frequently occurring width. (See 4.3.)

The coverage of a CPset is the total width of all the good CPs that it contains.

CPset A is better than CPset $\mathrm{B}$ if $\mathrm{A}$ has greater coverage, or equal coverage, but more good CPs, or equal good CPs, but more total CPs.

CPset A explains set B unless one or more of the following are true:

1. The edge of one of B's CPs lies outside of all of A's CPs. This is not allowed, as it shows that B has more text than $\mathrm{A}$.

2. The edges of one of B's CPs fall in different CPs of $\mathrm{A}$, and the width of the B CP is a common one. This means that A has split a column of common width.

3. The right edge of one of B's CPs falls in the same A $\mathrm{CP}$ as the left edge of the next B CP, and the B CPs are of roughly the same width. It looks like $\mathrm{A}$ has a different number of columns to $\mathrm{B}$. The same-width condition allows A to explain B with a pull-out.

4. Both edges of two CPs of B fall in the same CP of A. This means that A has merged two columns of B.

Note that the two edges of one of B's CPs are allowed to fall into two CPs of $\mathrm{A}$, as long as the width is not a common one. This allows headings that merge columns in B to be explained by A.

A list of column candidates is made from the set of CPsets on the page, ordered best first, and with duplicates eliminated by the A explains $B$ rules above. In

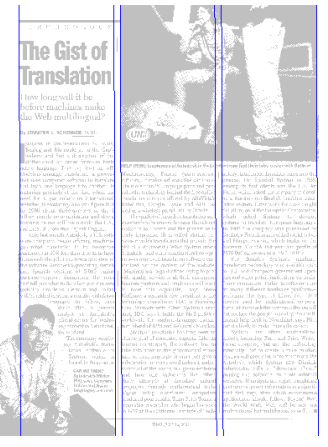

Fig.7. Columns. this process, all image CPs are ignored.

After the initial candidates are made, they are improved by adding new CPs and widening existing CPs, by using the edge of a CP in a different CPSet while widening doesn't cause overlap of CPs. 
An iterative process then labels the longest segment of consecutive (allowing for a very small region of failure) page y-coordinates that is explained by one of the column candidates. Fig. 7 shows the result of this process.

\section{Finding the regions}

After the columns are found, CPs are given a type according to how many columns they span. CPs within a single column are flowing, partitions that touch more than one column, but do not span to the outer edges of either are pull-out, and partitions that completely span more than one column are heading.

\subsection{Create flows of CPs}

Each $\mathrm{CP}$ chooses its best matching upper and lower partner, being the vertically nearest $\mathrm{CP}$ that overlaps horizontally. Since each CP registers itself with its chosen partner, each CP may have zero or more registered upper and lower partners.

The size of the list of registered partners is forced to become zero or one for each of upper and lower, using the following rules in order:

1. Type. If there are multiple types, text can only stay with its own (exact) type, whereas image can stay with any other image type.

2. Transitive partner shortcuts are broken. If $\mathrm{A}$ has 2 partners $\mathrm{B}$ and $\mathrm{C}$, and also $\mathrm{B}$ has $\mathrm{C}$ as a partner in the same direction, then delete $\mathrm{C}$ as a partner of $\mathrm{A}$, leaving a clean chain A-B-C. Also if A has a partner B, and B has a partner $\mathrm{A}$ in the same direction, break the cycle.

3. (Text only) If A still has 2 partners B, C, chase B and C's partners to see which has the longest chain. Delete from A the partner that has the shortest chain, and convert the type of the shortest chain to pull-out.

4. (Image only) Choose the partner $\mathrm{CP}$ with the largest horizontal overlap.

All CPs now have 0 or 1 partners. Even so, (re)run rule 1 above. This purifies all chains of text to a single type and splits text chains from image chains. Image chains are purified by setting all CPs in a chain to the most general type in the chain. Fig. 8 shows the final typed CPs, where flowing text is blue, heading text is cyan, heading image is magenta, and pullout image is orange.
Chains of text CPs are further divided into groups of uniform line-spacing, which make text blocks. Now each chain of CPs represents a candidate region, but the regions have to be ordered.

\subsection{Reading order determination}

Recall that image and text partitions are typed as one of 3 possibilities: flowing, pull-out, and heading. Also, the page is divided into sections of a consistent column layout. With this information, a reasonable reading order drops out of a few simple rules:

1. Flowing blocks follow by y position within a column.

2. Pull-out blocks follow by y position in an imaginary column between the real columns that they touch.

3. A heading spans multiple columns and follows anything that is above it in the columns spanned, or between them. Anything that lies in the same columns below the heading follows after it.

4. A change in column layout works just like a heading. Anything in any columns that are changed (or between them) goes before anything in the new columns. Unchanged columns are unaffected by a change in column layout.

5. Between headings, the content of columns is ordered from left to right.

\subsection{Find the polygon boundary for each region}

For simplicity of implementation, the region polygons are isothetic: i.e. edges alternate between being horizontal and parallel to the mean tab line (Approximately vertical.) The polygon edges are chosen to minimize the number of vertices, while satisfying the constraint that all CPs are contained within their region polygon, and no CP from another region intersects. Fig. 9 shows the final blocks created for the input image of Fig. 1.

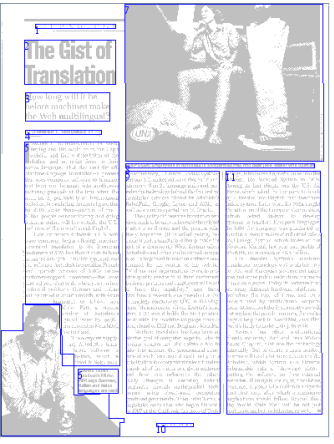

Fig.9. Final blocks.

\section{Testing and results}

The algorithm described herein is implemented in $\mathrm{C}++$, and the source code is available as part of the Tesseract open source OCR system $[9,10]$. It runs on a typical 8MPixel image in approximately 1 second on a 3.4 GHz Pentium 4. 


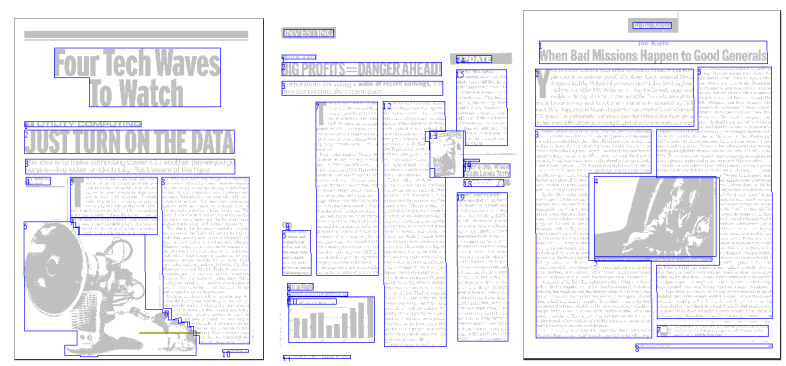

Fig.10. Results on some of the ICDAR2007 set.

Properly testing page layout analysis is a difficult problem [11] with very little publicly available groundtruth for complex magazine pages. The UNLV test set [12], only measures text regions, and counts errors unless figure captions are placed after all the body text.

The ICDAR page layout analysis competitions provide better measurement of overall accuracy, and the results of this algorithm appear in the 2009 competition [13]. Some graphical results are shown in Fig. 10 and numerical comparisons with the entrants in the ICDAR 2007 competition are shown in Table 1. The results in Table 1 are computed on only the 2007 test set, and the author would like to thank Apostolos Antonocopoulos for providing these results. For details on the testing methodology, see references [11] and [13].

Table 1. Results on the ICDAR 2007 set.

\begin{tabular}{|l|l|l|l|l|l|}
\hline Method & Noise & Sep & Text & Image & Overall \\
\hline \multicolumn{7}{|c|}{ PRImA Metric } \\
\hline $2007-$ Besus & $86.8 \%$ & $76.9 \%$ & $37.4 \%$ & $42.5 \%$ & $35.9 \%$ \\
\hline $2007-\mathrm{TH} 1$ & $68.0 \%$ & $79.7 \%$ & $76.1 \%$ & $46.2 \%$ & $67.6 \%$ \\
\hline $2007-\mathrm{TH} 2$ & $67.6 \%$ & $79.6 \%$ & $72.9 \%$ & $48.4 \%$ & $65.7 \%$ \\
\hline Tesseract & $65.6 \%$ & $74.1 \%$ & $72.1 \%$ & $55.3 \%$ & $68.4 \%$ \\
\hline \multicolumn{7}{|c|}{ F-Measure } \\
\hline $2007-$ Besus & $62.9 \%$ & $76.2 \%$ & $95.8 \%$ & $57.2 \%$ & $90.2 \%$ \\
\hline $2007-\mathrm{TH} 1$ & $79.2 \%$ & $80.7 \%$ & $91.9 \%$ & $72.1 \%$ & $88.2 \%$ \\
\hline $2007-\mathrm{TH} 2$ & $79.2 \%$ & $80.6 \%$ & $92.3 \%$ & $72.4 \%$ & $88.6 \%$ \\
\hline Tesseract & $79.2 \%$ & $70.9 \%$ & $93.3 \%$ & $82.0 \%$ & $91.3 \%$ \\
\hline \multicolumn{7}{|c|}{ Recall } \\
\hline $2007-$ Besus & $65.7 \%$ & $71.7 \%$ & $94.9 \%$ & $67.0 \%$ & $88.2 \%$ \\
\hline $2007-\mathrm{TH} 1$ & $65.6 \%$ & $79.5 \%$ & $96.9 \%$ & $66.4 \%$ & $89.8 \%$ \\
\hline $2007-\mathrm{TH} 2$ & $65.6 \%$ & $79.5 \%$ & $97.2 \%$ & $66.9 \%$ & $90.2 \%$ \\
\hline Tesseract & $65.6 \%$ & $81.4 \%$ & $97.9 \%$ & $76.5 \%$ & $93.8 \%$ \\
\hline \multicolumn{7}{|c|}{ Precision } \\
\hline $2007-$ Besus & $60.4 \%$ & $81.3 \%$ & $96.7 \%$ & $50.0 \%$ & $92.2 \%$ \\
\hline $2007-\mathrm{TH} 1$ & $100.0 \%$ & $81.9 \%$ & $87.4 \%$ & $79.0 \%$ & $86.7 \%$ \\
\hline $2007-\mathrm{TH} 2$ & $100.0 \%$ & $81.7 \%$ & $87.9 \%$ & $79.0 \%$ & $87.0 \%$ \\
\hline Tesseract & $100.0 \%$ & $62.8 \%$ & $89.0 \%$ & $88.3 \%$ & $88.9 \%$ \\
\hline
\end{tabular}

\section{Conclusion and further work}

Tab-stops make an interesting and useful alternative to white rectangles for finding the column structure of a page. Combining the top-down concept of column structure with bottom-up classification methods enables page layout analysis to easily handle the complex nonrectangular layouts of modern magazine pages without losing sight of the "bigger picture" that often happens when bottom-up methods are used alone.

The algorithm described has no table detection or analysis, but the tab-stops make particularly useful features for both, so table analysis will be added in the future.

\section{References}

[1] F. Wahl, K. Wong, R. Casey, "Block segmentation and text extraction in mixed text/image documents," Computer Graphics and Image Processing, 20, 1982, pp375-390.

[2] M. Chen, X. Q. Ding, "Unified HMM-based Layout Analysis Framework and Algorithm," SCI CHINA Ser F, 46(6), Dec. 2003, pp401-408.

[3] S.P. Chowdhury, S. Mandal, A.K. Das, B. Chanda, "Segmentation of Text and Graphics from Document Images," Proc. of the 9th Int. Conf. on Document Analysis and Recognition, IEEE, Curitiba, Brazil, Sep 2007, pp619623.

[4] G. Nagy, S.C. Seth, "Hierarchical Representation of Optically Scanned Documents" Proc. 7th Int. Conf. on Pattern Recognition, Montreal, Canada, 1984, pp347-349.

[5] H.S. Baird, S.E. Jones, S.J. Fortune, "Image Segmentation by Shape-directed Covers," Proc. 10th Int. Conference on Pattern Recognition, IEEE Atlantic City, NJ, 1990, pp820-825.

[6] T. Pavlidis, J. Zhou, "Page Segmentation and Classification," CVGIP: Graphical Models and Image Processing, 54(6), November 1992, pp484-496.

[7] T.M. Breuel, "Two Geometric Algorithms for Layout Analysis," Proc. of the 5th Int. Workshop on Document Analysis Systems V, Springer-Verlag 2002, pp188-199.

[8] Leptonica image processing and analysis library. http://www.leptonica.com.

[9] R. Smith. "An overview of the Tesseract OCR Engine." Proc $9^{\text {th }}$ Int. Conf. on Document Analysis and Recognition, IEEE, Curitiba, Brazil, Sep 2007, pp629-633.

[10] The Tesseract open source OCR engine. http://code.google.com/p/tesseract-ocr.

[11] A. Antonacopoulos, B. Gatos, D. Bridson, "ICDAR2007 Page Segmentation Competition," Proc 9 $9^{\text {th }}$ Int. Conf. on Document Analysis and Recognition, IEEE, Curitiba, Brazil, Sep 2007, pp1279-1283.

[12] UNLV ISRI OCR testing toolkit and database http://www.isri.unlv.edu/ISRI/OCRtk.

[13] A. Antonacopoulos et. al. "ICDAR2009 Page Segmentation Competition," Proc $10^{\text {th }}$ Int. Conf. on Document Analysis and Recognition, IEEE, Barcelona, Spain, Jul 2009. 